\title{
The actual Rees-Sciama effect from the local universe
}

\author{
M. Maturi ${ }^{1,2}$, K. Dolag ${ }^{2}$, A. Waelkens ${ }^{2}$, V. Springel ${ }^{2}$, and T. Enßlin ${ }^{2}$ \\ 1 Zentrum für Astronomie, ITA, Universität Heidelberg, Albert-Überle-Str. 2, 69120 Heidelberg, Germany \\ e-mail: maturi@ita.uni-heidelberg.de \\ 2 Max-Planck-Institute for Astrophysics, Karl-Schwarzschild-Str. 1, 85741 Garching, Germany
}

Received 14 August 2007 / Accepted 21 September 2007

\begin{abstract}
Observations of the Cosmic Microwave Background (CMB) have revealed an unexpected quadrupole-octopole alignment along a preferred axis pointing toward the Virgo cluster. We here investigate whether this feature can be explained in the framework of the concordance model by secondary anisotropies produced by the non-linear evolution of the gravitational potential, the so-called ReesSciama (RS) effect. We focus on the effect caused by the local superclusters, which we calculate using a constrained high-resolution hydrodynamical simulation, based on the IRAS 1.2-Jy all-sky galaxy redshift survey, which reproduces the main structures of our Universe out to a distance of $110 \mathrm{Mpc}$ from our Galaxy. The resulting RS effect peaks at low multipoles and has a minimum/maximum amplitude of $-6.6 \mu \mathrm{K} / 1.9 \mu \mathrm{K}$. Even though its quadrupole is well aligned with the one measured for the CMB, its amplitude is not sufficient to explain the observed magnitude of the quadrupole/octopole alignment. To have an effect comparable to the actual CMB fluctuations, photons traversing the local cosmic structures would need to experience a five/ten times larger gravitational redshift than would be expected in a standard scenario with dark matter and Newtonian gravity. In addition, we analyze the WMAP-3 data with a linear matched filter in an attempt to determine an upper limit for the RS signal amplitude on large scales. We found that it is possible to infer a weak upper limit of $30 \mu \mathrm{K}$ for its maximum amplitude.
\end{abstract}

Key words. cosmic microwave background

\section{Introduction}

The Wilkinson Microwave Anisotropy Probe (WMAP) observations of the Cosmic Microwave Background (CMB) (Spergel et al. 2003; Hinshaw et al. 2006) turned out to be in very good agreement with the predictions of the concordance $\Lambda$-CDM cosmology. However, the WMAP data also present a number of unexpected features at large angular scales. In particular, these include the small amplitude of the quadrupole (Spergel et al. 2003), as already noticed to be present within the COBE data by Hinshaw et al. (1996), the alignment between the CMB quadrupole and octopole, which are seen to point toward the direction of Virgo (Tegmark et al. 2003), the planarity of the octopole (de Oliveira-Costa et al. 2004), the alignment between the combined quadrupole and octopole momenta with the equinox and the ecliptic plane (Schwarz et al. 2004; Copi et al. 2006), the localized source of non-gaussianity (the cold spot) on angular scale $\sim 10^{\circ}$ (Vielva et al. 2004; Cruz et al. 2005), and the asymmetry of the large-scale power between the two galactic hemispheres (Eriksen et al. 2004; Hansen et al. 2004). The latter seems to be the most relevant one.

Different possible explanations for some of these features have been proposed, including a small universe with a cut-off scale below the cosmological horizon (Spergel et al. 2003), anisotropic universes such as Bianchi models (Bunn et al. 1996; Kogut et al. 1997; Jaffe et al. 2005), quintessence models (Dedeo et al. 2003) or local dust-filled voids (Inoue \& Silk 2006).

In the framework of the concordance $\Lambda$-CDM cosmology, we explore the possibility of explaining the above "anomalies" with the secondary $\mathrm{CMB}$ anisotropies produced by the evolution of the gravitational potentials of the local cosmological structures. These anisotropies are usually split into the integrated
Sachs-Wolfe (ISW) effect (ISW; Sachs \& Wolfe 1967; Hu \& Sugiyama 1994) and into the Rees-Sciama effect (RS; Rees \& Sciama 1968; Martinez-Gonzalez et al. 1990; Seljak 1996), which are produced by the linear and the non-linear evolution of the potential, respectively. We focus our attention on the RS effect although our simulation also includes the local ISW effect. However, the former is dominant over the latter in the volume we consider. We ignore distant structures beyond $110 \mathrm{Mpc}$, because their contribution to the RS effect is negligible at low multipoles (see for e.g. Seljak 1996; Cooray 2002).

A first study of the RS imprint from the local universe has been carried out by Cooray \& Seto (2005). They used a toymodel with two spherical mass concentrations of $8 \times 10^{15} h^{-1} M_{\odot}$ and $6.6 \times 10^{15} h^{-1} M_{\odot}$ to represent the Great Attractor and the Shapley concentration, and estimated a RS effect with a maximum amplitude of $\sim 0.5 \mu \mathrm{K}$. Similarly, Rakić et al. (2006) modeled the Shapley concentration with a spherical over-density (Lamaître-Tolman-Bondi model) with the Local Group falling into it. They estimate a RS signal which may amount to $\Delta T / T \sim$ $10^{-5}$ and conclude that the RS effect is not compatible with the features found in the WMAP data.

We use a high-resolution hydrodynamical simulation whose initial conditions were constrained to reproduce the density and velocity fields inferred with the IRAS 1.2-Jy all-sky galaxy redshift survey. The simulation gives a realistic picture of the local universe within a sphere of radius $110 \mathrm{Mpc}$ centered on our Galaxy. We use this simulation to obtain the expected RS effect on large scales in the full sky, and to define an optimal linear filter for measuring it in the WMAP-3 data.

The structure of the paper is as follows. In Sect. 2, we present the numerical simulation and the computation of the full sky map of the RS effect. In Sect. 3 we describe our analysis of the RS 


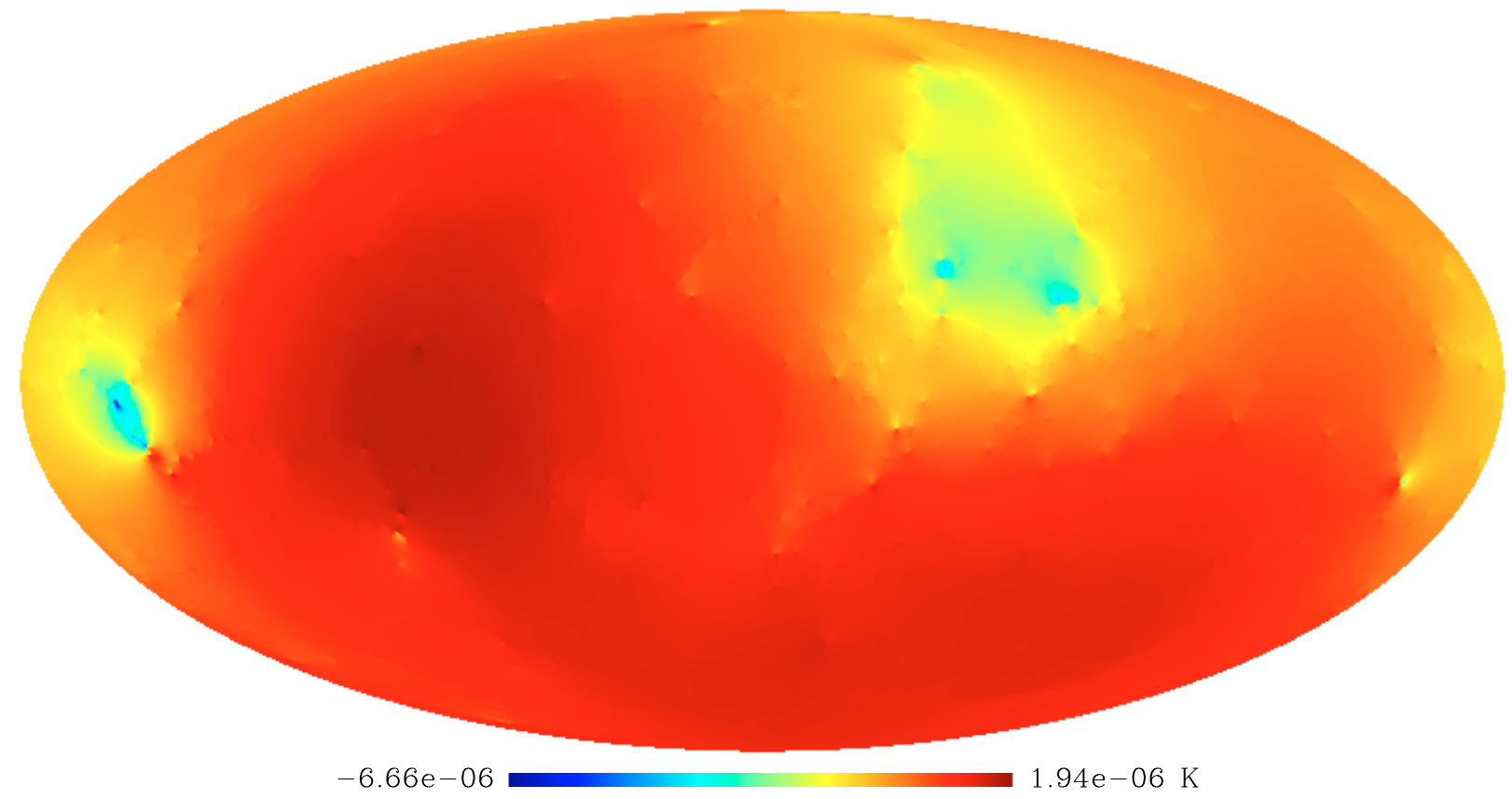

Fig. 1. Full sky map of the Rees-Sciama effect produced by the local universe within a sphere of radius $110 \mathrm{Mpc}$ centered on our Galaxy. This CMB secondary anisotropy is dominated by the gravitational collapse of the most massive structures present in our constrained high-resolution hydrodynamical simulation. These are the galaxy clusters of Virgo, A3637, Centaurus, Hydra and Perseus. The maximum signal strength corresponds to an induced temperature shift of $-6.6 \mu \mathrm{K}$ in the $\mathrm{CMB}$.

signal, and in Sect. 4 we propose a method based on a matched optimal filter to determine an upper limit for the amplitude of the RS effect in real data. Finally, we present our conclusions in Sect. 5.

\section{Simulating the RS effect due to the local universe}

\subsection{The numerical simulation}

The results presented in this paper have been obtained by using the $z=0$ output of a cosmological hydrodynamical simulation of the local universe. We used initial conditions similar to those adopted by Mathis et al. (2002) in their study (based on a pure $\mathrm{N}$-body simulation) of structure formation in the local universe. The galaxy distribution in the IRAS 1.2-Jy galaxy survey is first smoothed with a Gaussian filter on a scale of $7 \mathrm{Mpc}$ and then linearly evolved back in time up to $z=50$, following the method proposed by Kolatt et al. (1996). The resulting field is then used as a Gaussian constraint (Hoffman \& Ribak 1991) for an otherwise random realization of a flat $\Lambda$-CDM model, for which we assume a present matter density parameter $\Omega_{0}=0.3$, a Hubble constant $H_{0}=70 \mathrm{~km} \mathrm{~s}^{-1} \mathrm{Mpc}^{-1}$ and rms density fluctuations of $\sigma_{8}=0.9$ in spheres of comoving radius $8 h^{-1} \mathrm{Mpc}$, with $h=0.7$.

The volume that is constrained by the observational data covers a sphere of radius $\sim 110 \mathrm{Mpc}$ centered on the Milky Way. This region is sampled with more than 50 million high-resolution dark matter particles and is embedded in a periodic box of size $\sim 343 \mathrm{Mpc}$ on a side. The region outside the constrained volume is filled with nearly 7 million low-resolution dark matter particles, allowing a good coverage of long-range gravitational tidal forces that affect the high resolution region.

The analysis by Mathis et al. (2002) demonstrated that the evolved state of these initial conditions provides a good match to the density and velocity fields of the large-scale structure observed in the local universe. Unlike in the original simulation by Mathis et al. (2002), we however also follow the evolution of the gas distribution. To accomplish this we extended the initial conditions by splitting each of the original high-resolution particles into a gas and a dark matter particle, having masses of $0.48 \times 10^{9} M_{\odot}$ and $3.1 \times 10^{9} M_{\odot}$, respectively; this corresponds to a cosmological baryon fraction of 13 per cent. The total number of particles within the simulation is then slightly more than 108 million.

Our simulation run has been carried out with GADGET-2 (Springel 2005), a new version of the parallel Tree-SPH simulation code GADGET (Springel et al. 2001) with an entropyconserving formulation of SPH (Springel \& Hernquist 2002). The simulation neglected radiative cooling processes and employed a comoving gravitational force resolution (i.e. the comoving softening length) of $14 \mathrm{kpc}$ (Plummer-equivalent), which is comparable to the inter-particle separation reached by the SPH particles in the dense centers of our simulated galaxy clusters. Previously, the same simulation has been used to study the propagation of cosmic rays and to predict the SZ-effect from diffuse hot gas in the local universe (Dolag et al. 2005a,b).

In this work we neglect the contribution of all structures placed at a distance larger than $110 \mathrm{Mpc}$, because the volume covered by our simulation is sufficiently large to account for all relevant sources of the RS effect that contribute to the low multipoles we are interested in (see for e.g. Seljak 1996; Cooray 2002, where the total RS power spectrum is shown to peak at $30<l$ ). For example, the RS imprint of a galaxy cluster placed just outside the considered volume would subtend RS features with scales typically smaller than $\sim 0.5^{\circ}$, i.e. corresponding to multipoles larger than $l \sim 650$ (see for e.g. Maturi et al. 2007). We note that the RS effect of galaxy clusters can also be related to their gravitational lensing deflection field (Birkinshaw \& Gull 1983) whose typical scale is at most of the order of $1 \mathrm{Mpc}$. The inclusion of the neglected large volume beyond $110 \mathrm{Mpc}$ would be possible through larger numerical simulations (see for e.g. Springel et al. 2005) or through analytical modeling. However, a direct simulation approach would in general fail to reproduce the 
real matter distribution and dynamics, and hence fail to match with its induced RS effect the actual orientation of the low multipoles of the CMB.

\subsection{RS full sky map}

Photons change their energy when they traverse evolving gravitational potentials. This effect introduces secondary anisotropies in the CMB, which are usually split into two terms: the integrated Sachs-Wolfe effect (ISW, Sachs \& Wolfe 1967) and the Rees-Sciama effect (RS, Rees \& Sciama 1968), produced by the linear and the non-linear evolution of the potential, respectively. The resulting anisotropies can be calculated as

$$
\frac{\Delta T}{T}(\boldsymbol{\theta})=-\frac{2}{c^{3}} \int \dot{\Phi}(\boldsymbol{\theta} r, r / c) \mathrm{d} r,
$$

where $\dot{\Phi}$ refers to the time derivative of the gravitational potential, $\boldsymbol{\theta}$ is the position on the sky, $r$ is the space coordinate along the line-of-sight, and $r / c$ is the look-back time. These secondary temperature anisotropies provide precious information about the dynamical state and the evolution of the cosmic structures between the last scattering surface and us. In particular, the signal produced by the local universe could contribute to the low multipoles of the observed CMB anisotropies in such a way to partially explain the deviations from the expected Gaussian fluctuations as seen in the WMAP data.

In order to estimate the secondary anisotropies due to the local universe from our simulation we consider a Newtonian approximation where the gravitational potential is defined as

$\Phi(\boldsymbol{r}, t)=-G \int \mathrm{d}^{3} r^{\prime} \frac{\rho\left(\boldsymbol{r}^{\prime}, t\right)}{\left|\boldsymbol{r}-\boldsymbol{r}^{\prime}\right|}$,

so that its time derivative is

$\dot{\Phi}(\boldsymbol{r}, t)=-G \int \mathrm{d}^{3} r^{\prime} \rho\left(\boldsymbol{r}^{\prime}, t\right) \boldsymbol{v}\left(\boldsymbol{r}^{\prime}, t\right) \frac{\boldsymbol{r}-\boldsymbol{r}^{\prime}}{\left|\boldsymbol{r}-\boldsymbol{r}^{\prime}\right|^{3}}$,

where $\rho$ and $v$ are the matter density and velocity, respectively.

We directly solve Eq. (3) with a modified version of the parallel code GADGET-2 (Springel 2005), whose original version solves numerically a similar integral to compute the gravitational forces. The RS map is finally obtained by adopting the HEALPix pixelization of the sky (Górski et al. 2005) with parameter $n_{\text {side }}=512$, i.e. the pixels on the sky extend over $\sim 6.8^{\prime}$ on a side, and by evaluating Eq. (1) at each pixel position. We compute the integral along the line-of-sight as a direct sum by sampling $\dot{\Phi}$ at regular intervals of $140 \mathrm{~h}^{-1} \mathrm{kpc}$ between the radius $r_{\min }=1 \mathrm{Mpc}$ and $r_{\max }=110 \mathrm{Mpc}$. In doing so we include only the high resolution region of the numerical simulation in order to avoid any border effects. In this volume, the resolution is good enough to properly sample the RS signal down to the typical cluster core scale.

The resulting full-sky RS map due to the local universe is shown in Fig. 1. The main features are minima centered on those structures of the numerical simulation which correspond to the galaxy clusters of A3637, Virgo, Centaurus, Hydra and Perseus. The strongest of these signatures is related to Perseus (on the galactic equator, left hand side) with a negative peak of $-6.6 \mu \mathrm{K}$, while the structure corresponding to the cluster of Coma shows a very small contribution to the overall RS signal. The RS effects of other prominent clusters are visible, but their overall impact is negligible. The RS map slightly favors negative values because we sample a region of the universe populated by forming structures with an ongoing gravitational collapse.

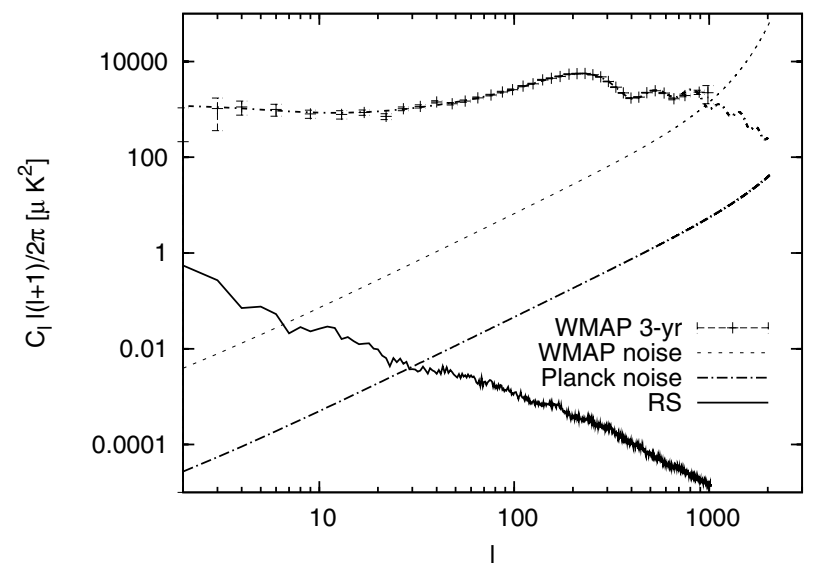

Fig. 2. Angular power spectra of the CMB, the RS effect from the local universe and the noise of WMAP $\left(20 \mu \mathrm{K}\right.$ and $\left.F W H M=30^{\prime}\right)$ and of Planck $\left(10 \mu \mathrm{K}\right.$ and $\left.F W H M=5^{\prime}\right)$. The RS effect is well below the amplitude of the primary CMB, but for a few low multipoles it is larger than the instrumental noise level.

Table 1. Amplitude of the multipoles $l=1, l=2$ and $l=3$ of the RS effect as derived in our simulation, and of the CMB as measured by the 3 year WMAP data (Hinshaw et al. 2006).

\begin{tabular}{lll}
\hline \hline & WMAP & $\mathrm{RS}$ \\
\hline$l=1$ dipole & removed & $\Delta T_{l=1}=0.72 \mu \mathrm{K}$ \\
$l=2$ quadrupole & $\Delta T_{l=2}=14.5^{+15} \mu \mathrm{K}$ & $\Delta T_{l=2}=0.74 \mu \mathrm{K}$ \\
$l=3$ octopole & $\Delta T_{l=3}=32_{-8}^{+26} \mu \mathrm{K}$ & $\Delta T_{l=3}=0.51 \mu \mathrm{K}$ \\
\hline
\end{tabular}

\section{Power spectrum analysis of the RS map}

The power spectra of our RS map, of the primary CMB, and of the instrumental noise of WMAP and of the upcoming Planck experiment are shown in Fig. 2. The RS signal is dominated by large-scale features which are only above the instrumental noise level for a few multipoles $(l<6$ for WMAP and $l<30$ for Planck). However, the RS signal is always well below the CMB primary fluctuations even on the largest scales where our covered volume should be sufficient to give the full expected signal. A detailed comparison between the amplitudes of the dipole, quadrupole and octopole of the RS map and of the CMB, as observed in the 3-years WMAP data (Hinshaw et al. 2006), is given in Table 1. It is curious to notice the similar alignment of the quadrupole and octopole components of the RS and of the $\mathrm{CMB}$, see Fig. 3. But in any case, the RS effect from the local universe is far from being a relevant contribution to the CMB anisotropies because of its small amplitude, which corresponds only to a contribution at the percent level. To explain the observed CMB quadrupole and octopole features, photons traversing the local cosmic structures would need to feel a five/ten times larger gravitational redshift than would be expected in a standard scenario with dark matter and Newtonian gravity.

We conclude that in Newtonian gravity the RS effect due to the local universe does not explain the deviations of the CMB from the expected primary anisotropies predicted by the concordance $\Lambda$-CDM model, i.e. the non-Gaussian features and the quadrupole-octopole alignment. However, we also found that the RS amplitude lies above the instrumental noise level for low $l$, and thus it may be possible to determine an upper limit for it, even if very weak, thanks to the present or upcoming full sky CMB experiments. On one hand a detection would help to understand the local cosmological structures and the details of their formation. On the other hand, if it was impossible to achieve a 
detection with an optimally designed filter then this would effectively ensure that the RS effect from the local universe constitutes a negligible foreground for any kind of data analysis of the $\mathrm{CMB}$ experiments. In the next section, we therefore explore the feasibility of measuring the RS signal from CMB data by using a linear matched filter defined on the sphere.

\section{Measuring the RS effect}

We wish to construct a linear matched filter allowing the RS effect from the local universe to be extracted from millimetric observations of the CMB. We want the filter to suppress, as well as possible, the primary anisotropies of the CMB as well as the instrumental noise. In this work we ignore other possible sources of noise such us the residuals of the galactic foreground separation. Because of the significant level of such contaminations of the CMB primary anisotropies we only expect to obtain an upper limit constraint.

\subsection{Non symmetric matched filter on the sphere}

The filter used in this work is based on a template for the expected signal and on the power spectrum of the noise. It was proposed by Haehnelt \& Tegmark (1996) in a flat sky approximation to estimate the peculiar velocities of galaxy clusters through their kinetic Sunyaev-Zel'dovich effect. Subsequently, it has also been proposed as a tool to estimate the mass of galaxy clusters through weak lensing of the CMB (Seljak \& Zaldarriaga 2000) and for cluster detection in optical weak lensing surveys (Maturi et al. 2005). An extension of the filter to the sphere was first introduced by Schaefer et al. (2004), assuming axially symmetric models for the expected signal.

We generalize the filter on the sphere for any non-axially symmetric model which, like the RS effect, has a known orientation and position. Thus, unlike in Schaefer et al. 2004, where the position of the signal is not previously known, we do not need to probe all orientations by performing a time consuming full sky convolution. We model the measured data $d(\boldsymbol{\theta})$, which contains a signal $s(\boldsymbol{\theta})=A \tau(\boldsymbol{\theta})$ contaminated by some noise $n(\boldsymbol{\theta})$, by

$d(\boldsymbol{\theta})=s(\boldsymbol{\theta})+n(\boldsymbol{\theta})=A \tau(\boldsymbol{\theta})+n(\boldsymbol{\theta})$.

In our case, $n(\boldsymbol{\theta})$ is mainly given by the sum of the CMB primary anisotropies and the instrumental noise. Both can be sufficiently well approximated with Gaussian random fields with zero mean. The noise correlation function is

$\left\langle n_{l m} n_{l^{\prime} m^{\prime}}^{*}\right\rangle=C_{l} \delta_{l, l^{\prime}} \delta_{m, m^{\prime}}$,

where the asterisk denotes the complex conjugate, $\delta$ is the delta function, and $C_{l}=C_{l, \mathrm{cmb}}+C_{l, \text { inst }}$ is the total angular power spectrum of the noise.

For convenience, all quantities are derived in the harmonic space. We wish to construct a linear filter $\Psi(\theta)$ which yields an estimate $\hat{A}$ for the amplitude $A$ of the signal, viz.

$\hat{A}=\sum_{l=0}^{+\infty} \sum_{m=-l}^{l} d_{l m} \Psi_{l m}^{*}$,

where $d_{l m}$ and $\Psi_{l m}$ are the harmonic coefficients of the data and of the filter function, respectively. The sum over $l$ is usually restricted to the maximum multipole given by the resolution of the observations.
The estimate is required to be unbiased and optimal, i.e. the quantity

$b \equiv\langle\hat{A}-A\rangle=A\left[\sum_{l=0}^{+\infty} \sum_{m=-l}^{l} \tau_{l m} \Psi_{l m}^{*}-1\right]$,

has to vanish and the rms of the measurement

$\sigma^{2} \equiv\left\langle(\hat{A}-A)^{2}\right\rangle=b^{2}+\sum_{l=0}^{+\infty} \sum_{m=-l}^{l} C_{l}\left|\Psi_{l m}\right|^{2}$,

has to be minimal. The filter $\Psi$ which satisfies these two conditions is the one which minimizes the action $L=\sigma^{2}+\lambda b$, where $\lambda$ is a Lagrange multiplier. We thus obtain

$\Psi_{l m}=\left[\sum_{l=0}^{+\infty} \sum_{m=-l}^{l} \frac{\left|\tau_{l m}\right|^{2}}{C_{l}}\right]^{-1} \frac{\tau_{l m}}{C_{l}}$

This shows that the filter is most sensitive for those spatial frequencies where the signal $\tau$ is large and the noise $C_{l}$ is small. Note that this filter uses the full information available on the expected signal $\tau$ and not only its power spectrum, as for example a Wiener filter would do.

\subsection{An upper limit for the RS amplitude in the WMAP-3 data}

Before applying our procedure to real data, we have tested the described filter on mock data where we combined the full sky RS map (see Sect. 2.2) from our simulation of the local universe with a realization of the primary CMB fluctuations computed with CMBEASY (Doran 2003) for a standard WMAP-3 cosmology. The resulting map was then degraded by adding an instrumental noise with the power spectrum

$C_{l, \text { inst }}=w^{-1} \exp \left[\frac{l(l+1) \theta_{F W H M}^{2}}{8 \ln 2}\right]$,

where $w^{-1}:=\left(\sigma_{T} \theta_{F W H M}\right)^{2}($ Knox 1995). The resolution and sensitivity were fixed according to the characteristics of the WMAP satellite $\left(\theta_{F W H M}=30^{\prime}, \sigma_{T}=20 \mu \mathrm{K}\right)$ and to the upcoming ESA space mission Planck $\left(\theta_{F W H M}=5^{\prime}, \sigma_{T}=10 \mu \mathrm{K}\right)$. A convolution with a Gaussian kernel with the same $F W H M$ is applied to the simulated data. The dipole component of the signal is finally removed because of the strong contamination which would be caused by the proper motion of the solar system. We ignore other contaminants such as residuals of the foreground subtraction or extragalactic point sources.

The resulting map is finally analyzed with the filter described in Sect. 4.1. For the filter template $\tau$ we use the same RS map which was also included in the simulated observations. This is a strong simplification, but here we only aim at testing the performance of the filter when all the conditions for its application are optimally satisfied. The results are as follows:

$$
\begin{array}{lll}
\text { Expected signal } & : & A=1.9 \mu \mathrm{K} \\
\text { WMAP (simulation) } & : & A=3.4 \pm 13 \mu \mathrm{K} \\
\text { Planck (simulation) } & : & A=2.2 \pm 12 \mu \mathrm{K} .
\end{array}
$$

It is thus only possible to infer a weak upper limit for the amplitude of the RS effect due to the local universe, because of the strong contamination given by the CMB primary fluctuations.

Finally, we apply the filter, as previously described, to the 3years ILC map of the WMAP experiment (Hinshaw et al. 2006). 

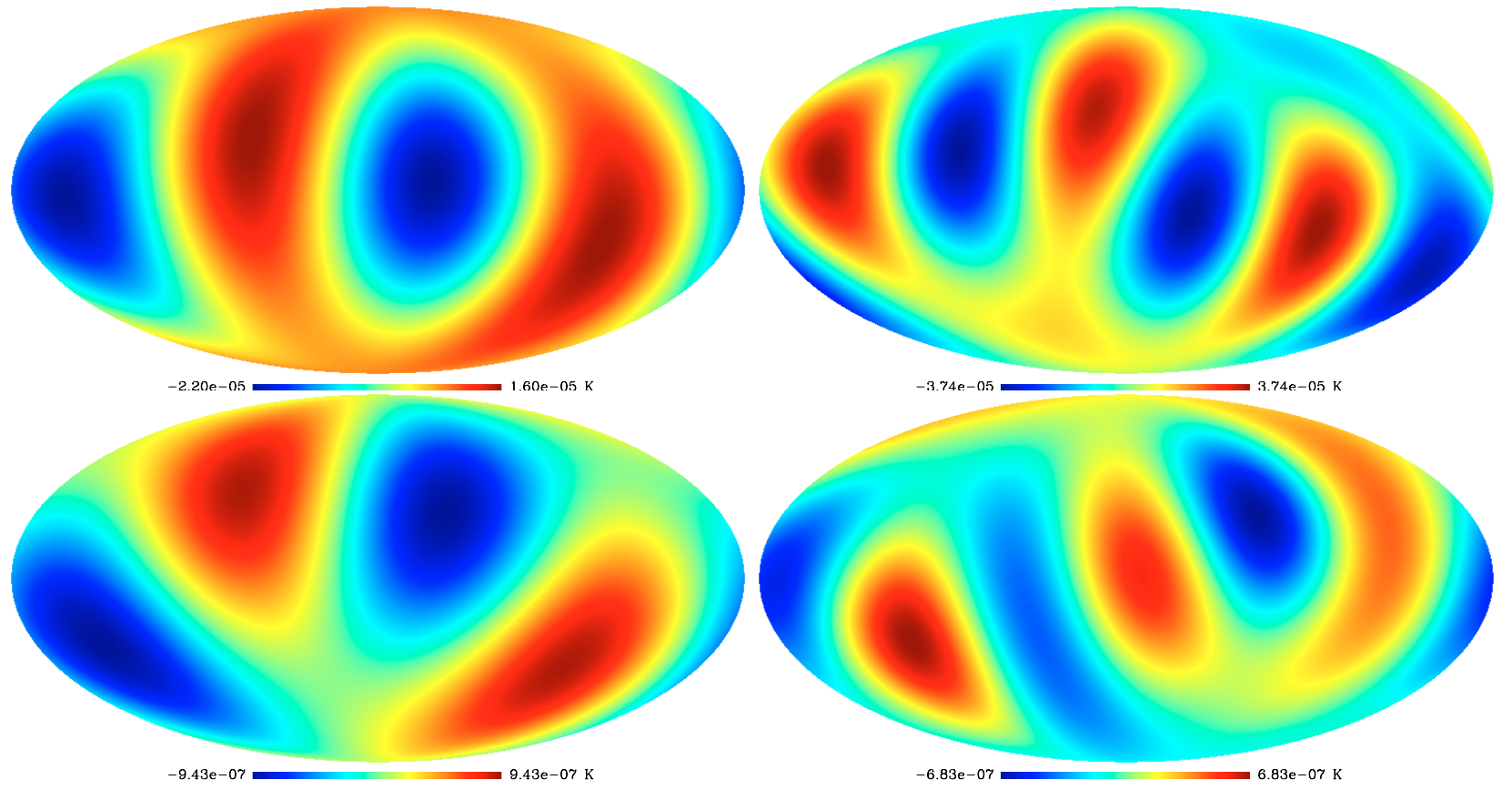

Fig. 3. Comparison between the quadrupole and the octopole of the CMB as measured by WMAP (top panels) and of the RS effect from the local universe (bottom panels).

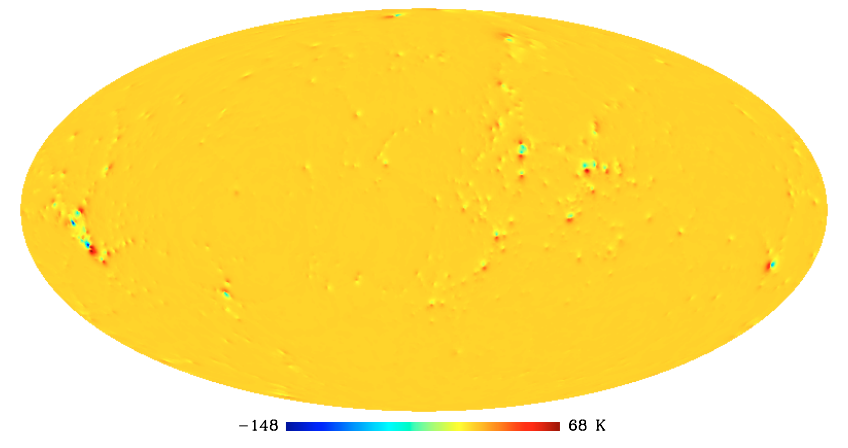

Fig. 4. Filter function used to estimate the upper limit for the maximum amplitude of the RS effect produced by the local universe.

In this case, since the ILC map is convolved with a Gaussian kernel with $F W H M=1^{\circ}$, we convolved the template $\tau$ in the same way before the filter derivation. This convolution of the template has the implicit advantage of suppressing small features in the RS template which may not be present in the structures of the real universe. The resulting filter is shown in Fig. 4.

To estimate the variance of the measurement, we refrain from using Eq. (8) because it supposes that the template is identical to the expected signal and thus Eq. (8) would yield a lower limit for the noise amplitude. We rather estimate the noise level by applying the filter many times with the RS template randomly rotated on the unit sphere. While the mean of these randomized measurements should be consistent with zero because the rotated templates do not match the expected signal, the dispersion around zero provides a good estimate of the noise level. We obtain

WMAP (ILC map) : $\quad A=3 \pm 26 \mu \mathrm{K}$

which implies an upper limit for the amplitude of the RS effect in the actual CMB data of $A_{\max }=30 \mu \mathrm{K}$. This is not very restrictive compared to the expected signal strength of $A_{\text {exp }}=1.9 \mu \mathrm{K}$.

\section{Conclusions}

We estimated a realistic map of the Rees-Sciama effect causd by the evolution of the gravitational potential in the local universe within a sphere with a radius of $110 \mathrm{Mpc}$ centered on our Galaxy. To this end we used a high-resolution hydrodynamical simulation whose initial conditions were constrained to reproduce the density and velocity fields inferred from the IRAS 1.2-Jy all-sky galaxy redshift survey.

We estimated a minimum/maximum amplitude of the local RS effect of $-6.6 \mu \mathrm{K} / 1.9 \mu \mathrm{K}$, which is a factor of 10 larger than the $0.5 \mu \mathrm{K}$ predicted by Cooray \& Seto (2005). The power spectrum of the RS signal lies above the instrumental noise of WMAP and Planck for low multipoles but is always well below the CMB primary anisotropies. Interestingly, the RS signal's quadrupole and octopole moments are well aligned with those of the CMB primary fluctuations. However, the small amplitudes of these RS multipoles are not sufficient to explain the quadrupoleoctopole alignment observed by COBE and WMAP in the CMB.

In addition, we attempted to measure the RS effect on large scales in mock CMB observations as well as in real data using a matched filter technique. We found that it is only possible to place a rather weak upper limit for its amplitude even under optimistic assumptions. In analyzing the 3-years ILC map of WMAP (Hinshaw et al. 2006) this upper limit turned out be $A_{\max }=30 \mu \mathrm{K}$, which is not very restrictive compared with the expected signal of $A_{\text {exp }}=1.9 \mu \mathrm{K}$.

\section{References}

Birkinshaw, M., \& Gull, S. F. 1983, Nature, 302, 315

Bunn, E. F., Ferreira, P. G., \& Silk, J. 1996, Phys. Rev. Lett., 77, 2883

Cooray, A. 2002, Phys. Rev. D, 65, 083518

Cooray, A., \& Seto, N. 2005, J. Cosmol. Astro-Part. Phys., 12, 4

Copi, C. J., Huterer, D., Schwarz, D. J., \& Starkman, G. D. 2006, MNRAS, 367, 79

Cruz, M., Martínez-González, E., Vielva, P., \& Cayón, L. 2005, MNRAS, 356, 29

de Oliveira-Costa, A., Tegmark, M., Zaldarriaga, M., \& Hamilton, A. 2004, Phys. Rev. D, 69, 063516

Dedeo, S., Caldwell, R. R., \& Steinhardt, P. J. 2003, Phys. Rev. D, 67, 103509 
Dolag, K., Grasso, D., Springel, V., \& Tkachev, I. 2005a, J. Cosmol. Astro-Part. Phys., 1, 9

Dolag, K., Hansen, F. K., Roncarelli, M., \& Moscardini, L. 2005b, MNRAS, 363,29

Doran, M. 2003, [arXiv: astro-ph/0302138]

Eriksen, H. K., Hansen, F. K., Banday, A. J., Górski, K. M., \& Lilje, P. B. 2004, ApJ, 605, 14

Górski, K. M., Hivon, E., Banday, A. J., et al. 2005, ApJ, 622, 759

Haehnelt, M. G., \& Tegmark, M. 1996, MNRAS, 279, 545

Hansen, F. K., Balbi, A., Banday, A. J., \& Górski, K. M. 2004, MNRAS, 354, 905

Hinshaw, G., Banday, A. J., Bennett, C. L., et al. 1996, ApJ, 464, L17

Hinshaw, G., Nolta, M. R., Bennett, C. L., et al. 2006, ArXiv Astrophysics e-prints

Hoffman, Y., \& Ribak, E. 1991, ApJ, 380, L5

Hu, W., \& Sugiyama, N. 1994, Phys. Rev. D, 50, 627

Inoue, K. T., \& Silk, J. 2006, ApJ, 648, 23

Jaffe, T. R., Banday, A. J., Eriksen, H. K., Górski, K. M., \& Hansen, F. K. 2005, ApJ, 629, L1

Knox, L. 1995, Phys. Rev. D, 52, 4307

Kogut, A., Hinshaw, G., \& Banday, A. J. 1997, Phys. Rev. D, 55, 1901

Kolatt, T., Dekel, A., Ganon, G., \& Willick, J. A. 1996, ApJ, 458, 419

Martinez-Gonzalez, E., Sanz, J. L., \& Silk, J. 1990, ApJ, 355, L5
Mathis, H., Lemson, G., Springel, V., et al. 2002, MNRAS, 333, 739

Maturi, M., Meneghetti, M., Bartelmann, M., Dolag, K., \& Moscardini, L. 2005, A\&A, 442, 851

Maturi, M., Enßlin, T., Hernández-Monteagudo, C., \& Rubiño-Martín, J. A. 2007, A\&A, 467, 411

Rakić, A., Räsänen, S., \& Schwarz, D. J. 2006, MNRAS, 369, L27

Rees, M. J., \& Sciama, D. W. 1968, Nat, 217, 511

Sachs, R. K., \& Wolfe, A. M. 1967, ApJ, 147, 73

Schaefer, B. M., Pfrommer, C., Hell, R., \& Bartelmann, M. 2004, [arXiv: astro-ph/0407090]

Schwarz, D. J., Starkman, G. D., Huterer, D., \& Copi, C. J. 2004, Phys. Rev. Lett., 93, 221301

Seljak, U. 1996, ApJ, 460, 549

Seljak, U., \& Zaldarriaga, M. 2000, ApJ, 538, 57

Spergel, D., Verde, L., Peiris, H., Komatsu, E., et al. 2003, ApJS, 148, 175

Springel, V. 2005, MNRAS, 364, 1105

Springel, V., \& Hernquist, L. 2002, MNRAS, 333, 649

Springel, V., Yoshida, N., \& White, S. 2001, New Astron., 6, 79

Springel, V., White, S. D. M., Jenkins, A., et al. 2005, Nature, 435, 629

Tegmark, M., de Oliveira-Costa, A., \& Hamilton, A. J. 2003, Phys. Rev. D, 68, 123523

Vielva, P., Martínez-González, E., Barreiro, R. B., Sanz, J. L., \& Cayón, L. 2004, ApJ, 609, 22 